\title{
Altered gut microbiome promotes proteinuria in mice induced by Adriamycin
}

\author{
Qian Jiang ${ }^{1,3}$, Xiwei He ${ }^{2}$, Yuntao Zou ${ }^{3}$, Yin Ding ${ }^{3}$, Huang $\mathrm{Li}^{1^{*}}$ and Huimei Chen ${ }^{3,4}$
}

\begin{abstract}
Inflammation has recently been attributed to dysbiosis of the gut microbiome, which has been linked to proteinuria in chronic kidney disease. Since Adriamycin ${ }^{\circledR}$ (ADR) is widely used to induce proteinuria in mouse models, the aim of this study was to explore the potential effect of gut microbiome on this process. Both ADR resistant (C57BL/6) and susceptible (BALB/C) strains were part of the induced nephropathy with ADR injection. BALB/C mice significantly presented increased urinary albumin/creatinine ratio (UACR) with renal lesions in pathology, but C57BL/6 mice were absent from kidney damage. Species and genus level resolution analysis showed a shift in gut microbial profile between BALB/C and C57BL/6 mice. ADR further altered the stool microbiome in BALB/C mice, particularly with enrichment of Odoribacter and depletion of Turicibacter, Marvinbryantia and Rikenella. Moreover, the level of UACR in BALB/C mice was marked related to the abundance of Marvinbryantia, Odoribacter and Turicibacter in stool. Meanwhile, ADR remarkably increased the serum levels of interleukin (IL)-2 in BALB/C mice, but not in C57BL/6 mice. It is suggested that the favorably altered stools as shown in the microbiome might promote the inflammation and proteinuria in ADR-sensitive mice, which provides a new insight on the pathogenicity of chronic kidney disease.
\end{abstract}

Keywords: Gut microbiome, Proteinuria, Chronic kidney disease, Adriamycin

\section{Introduction}

Chronic kidney disease (CKD) is a major public health problem that has received increasing attention because of its association with the high rate of end stage renal disease (ESRD) (Eknoyan et al. 2004; Liu et al. 2015; Martinez-Castelao et al. 2014). Proteinuria is often observed in patients with CKD, and it is reportedly a vital risk factor for the progression and prognosis of CKD (Sandsmark et al. 2015). Infections, toxins, diabetes and high blood pressure have been investigated in the pathogenesis of proteinuria and glomerulosclerosis. The status of inflammation is also suggested to be involved in proteinuria development. However, the source and alternation of inflammation in CKD has been ignored, especially in those patients without infection, auto-immunology disease and allergic disease.

*Correspondence: lihuang76@nju.edu.cn

1 Orthodontic Department, Nanjing Stomatological Hospital, Medical

School of Nanjing University, Nanjing, Jiangsu, China

Full list of author information is available at the end of the article
Recently, an increased interest in the role of gut microbiome has gained momentum and increasing evidence supports a kidney-gut axis (Evenepoel et al. 2016; Vaziri et al. 2013). The dysbiosis of the gut microbiota was observed in CKD, which produces key neurovascular toxins, including indoxyl sulphate (IS) and $p$-cresyl sulphate (PCS) (Evenepoel et al. 2016; Okuda et al. 1986). Moreover, these toxins and their associations would increase inflammation, kidney disease progression and then cause mortality in the CKD population (Evenepoel et al. 2016; Stenvinkel 2005; Vaziri et al. 2013). There is a need for conclusive findings that can elucidate whether there is a causal role of gut microbiome in proteinuria.

To test the hypothesis that the dysbiosis in the gut microbiome accompanied by inflammation can cause proteinuria, we analyzed the gut microbiome profile in the Adriamycin (ADR)-induced nephropathy in BALB/C mice, which was an ADR susceptible strain, as well C57BL/6, an ADR resistant strain that served as a control. The correlations of significantly altered microbes with urine protein levels and the levels of inflammation were demonstrated. It suggested an altered stool 
microbiome might promote the inflammation and proteinuria in ADR-sensitive mice.

\section{Materials and methods Experimental animals}

6-week-old mice with inbred strains of BALB/C and C57BL/6 were obtained from the experimental animal center of the Academy of Military Medical Science of China. All animal study protocols were approved by the Vanderbilt University Institutional Animal Care and Use Committee. Male BALB/C mice were divided into two groups with six animals in each, so were C57BL/6. Mice were housed in a specific pathogen free environment with temperature- and humidity-controlled environment under $12 \mathrm{~h}$ light $/ 12 \mathrm{~h}$ dark conditions. They were fed a standard rodent diet in accordance with our institutional guidelines for the care and use of laboratory animals.

To establish the animal model of ADR-induced nephropathy, BALB/C and C57BL/6 mice received a single intravenous injection of ADR $(10.0 \mathrm{mg} / \mathrm{kg}$; Sigma, St. Louis, MO). Control mice were treated with an equivalent intravenous volume of normal saline (NS). The urinary samples were individually collected using metabolism cages at $0 \mathrm{~h}, 1$ and 2 weeks, and the mice were killed at 2 weeks after ADR or NS injection. Cardiac blood, urine, stool and kidney tissue were collected for analysis. Nine C57BL/6 and BALB/C male mice were used in each group. ADR injection duration was varied from $24 \mathrm{~h}$ to 7 weeks with an optimal dose of $10-11 \mathrm{mg} /$ kg body wt of ADR (Sun et al. 2013; Wang et al. 2000). In BALB/C mice, glomerular endothelial cells undergoing apoptosis could be detected as early as $24 \mathrm{~h}$ after ADR administration (Sun et al. 2013). While the overt proteinuria was reported to appear at day 5 and was maximal at day 7 (Wang et al. 2000). Therefore, we chose 1 and 2 weeks after ADR treatment for blood, urine and kidney tissue collection.

\section{Measurements of serum or urine parameters}

The serum was separated by allowing the whole blood to stand at room temperature for $2 \mathrm{~h}$. Creatinine concentrations were measured using a Creatinine (CREA) reagent (Shanghai Gaozong Medical Technology Co, Shanghai, China) and urine albumin concentrations were determined using the BCA Protein Assay Kit (Thermo Scientific, Waltham, MA, USA). CCr (creatinine clearance) was calculated according to a Cockcroft-Gault formula: urinary volume $(\mathrm{ml}) \times$ urinary creatinine $(\mathrm{mg} / \mathrm{dl})) /($ plasma creatinine $(\mathrm{mg} / \mathrm{dl}) \times$ urine collection length (min) (Hsu et al. 2016). Results were expressed as $\mathrm{ml} / \mathrm{min} / 100 \mathrm{~g}$ body weight. The urinary albumin-tocreatinine ratio (UACR) in $\mathrm{g} / \mathrm{mol}$ was calculated as the urinary albumin concentration divided by the creatinine concentration.

Interleukin-2 (IL-2) levels in serum were determined by ELISA. ELISA assays were carried out using an immunoassay kit-Mouse IL-2 Quantikine ELISA Kit M2000, (R\&D Systems, Minneapolis, MN) according to the manufacturer's protocol.

\section{Histological analysis of kidney}

Mouse kidney samples were fixed in $10 \%$ formaldehyde, embedded in paraffin and cut into $4 \mu \mathrm{m}$ sections. Each section was routinely stained with H\&E. The pathological changes were observed using a light microscope and photographs were obtained. Sections were examined by a qualified pathologist who was blinded to other data. 10 images were captured and scored for each sample. The pathologist's job was to evaluate the degree of pathological changes based on glomerular epithelial hyperplasia.

\section{High-throughput sequencing of fecal DNA}

Genomic DNA from fecal samples (50-100 mg) was extracted using a FastDNA SPIN kit for soil (MP Biomedicals, CA, USA). The concentrations and purity of the resultant DNA were determined by a NanoDrop (NanoDrop ND-2000, USA) and stored at $-80{ }^{\circ} \mathrm{C}$ for further analysis.

The 16S rRNA gene in the fecal DNA samples was amplified by polymerase chain reaction (PCR) with primers 16s-F (5'-AGAGTTTGATYMTGGCTCAG- $3^{\prime}$ ) and 16s-R (5'-TGCTGCCTCCCG TAGGAGT-3') targeting the hypervariable $\mathrm{V} 1-\mathrm{V} 2$ region of the $16 \mathrm{~S}$ rRNA gene of bacteria. PCR products were purified using a DNA Fragment Purification Kit (Takara, Japan), and then barcoded and pooled to construct the sequencing library. An Illumina Mi-seq (Illumina, USA) was used for sequencing to generate 150,150 reads. The sequences obtained were deposited in a NCBI Sequence Read Archive under accession number PRJNA327732.

\section{Post-run analysis}

After sequencing, the raw data was sorted into different samples according to the barcodes. A sickle tool was applied to perform quality filtering of the raw reads and the quality was greater than 20 without any unknown bases (Joshi and Fass 2011). The modified pipeline (http://www.mothur.org/wiki/MiSeq_SOP) was used for the bioinformatics analysis and the number of the sequences was normalized by randomly extracting 29,966 reads to fairly compare all the samples at the same sequencing depth.

'Aligner' and 'Complete Linkage Clustering' were applied to calculate the operational taxonomic units (OTUs) with a threshold of $97 \%$ sequence similarity. 
The Chao index was then calculated using Mothur. Taxonomic classification of each sample was individually conducted using Ribosomal Database Project (RDP) Classifier (version 2.6) with a confidence threshold of $80 \%$. Cluster analysis was conducted with paleontological statistics software (PAST, version 3.01). The heat map of bacteria genus was conducted with $\mathrm{R}$ Language (version 3.1).

\section{Statistical analysis}

All data were presented as mean \pm SEM and analyzed using SPSS version 20.0. Comparisons were performed using an independent $t$ test for two groups or ANOVA (one-way analysis of variance) for four groups when parameters showed a normal distribution; otherwise it was performed using non-parametric tests. Repeatedmeasures ANOVA was used to test the difference of weight, $\mathrm{CCr}$ and UACR for different groups at three time points. The sequence number data of gut microbiome were normalized by $\log 10$ transformations prior to correlation analysis. The association between proteinuria and gut microbiome was revealed by bivariate correlation analysis and logistic regression analysis. Differences were considered significant at $p<0.05$.

\section{Results \\ $B A L B / C$ mice were sensitive to ADR}

Before ADR injection, BALB/C mice presented similar levels of body weight $(\mathrm{p}=0.543)$ and $\mathrm{CCr}(\mathrm{p}=0.824)$, but higher levels of UACR $(\mathrm{p}=0.003)$ when compared with C57BL/6 mice (Fig. 1a-c). The ADR injection further enhanced the difference of UACR between BALB/C and C57BL/6 mice $(\mathrm{p}<0.001)$. After ADR injection, the UACR levels were significantly increased by twofold $(\mathrm{p}<0.001)$ in BALB/C mice. ADR also lead to histological lesions in BALB/C mice. Figure 1d shows mesangial proliferation and segmental glomerular sclerosis in glomeruli in the BALB/C ADR group. However, ADR had no impact on $\mathrm{C} 57 \mathrm{BL} / 6$ mice at body weight, $\mathrm{CCr}$ and UACR.

\section{Gut microbiome altered with ADR in BALB/C mice}

Gut microbiome were determined by Illumina $\mathrm{Mi}$ seq sequencing using stool samples. Alpha diversity

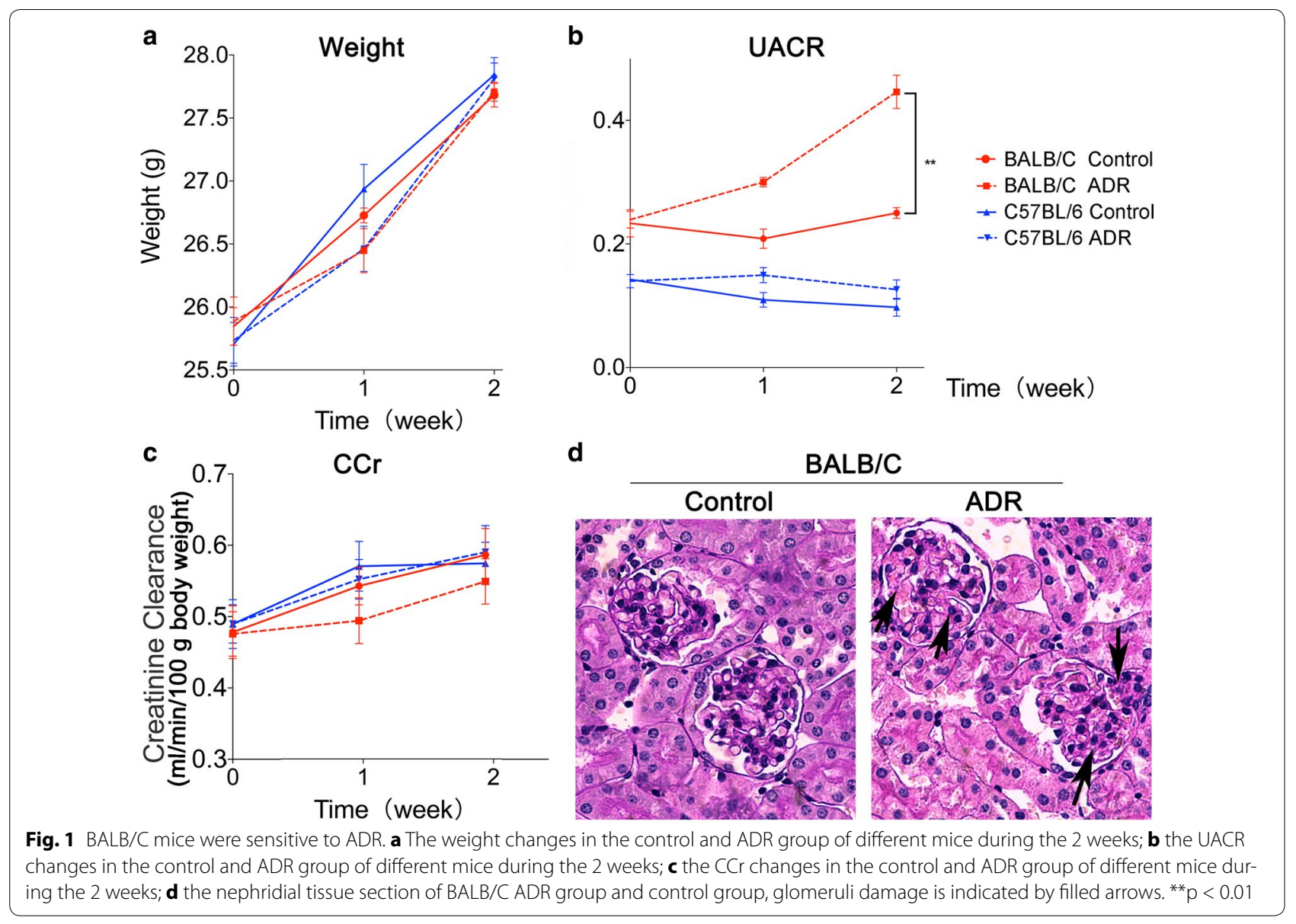


in BALB/C and C57BL/6 mice was $2683.54 \pm 492$ and $2897.85 \pm 244$, respectively $(p=0.75$, Fig. $2 a$ ). A slight decrease of microbiome diversity was observed after ADR exposure, but it did not reach the statistical significance (both $\mathrm{p}>0.05$ ). At phylum level, Bacteroidetes (52-61\%) and Firmicutes (32-42\%) were predominant and followed by Proteobacteria (3-6\%), Actinobacteria (1-2\%) and Tenericutes (0-2\%) (Fig. 2b). There was no significant difference between the two kinds of mice, and ADR injection did not obviously alter the phylum distribution, either.

Figure 3 shows an interesting separation of the top 1\% genera between BALB/C and C57BL/6 mice. Compared with $\mathrm{C} 57 \mathrm{BL} / 6$ mice, BALB/C presented higher abundance of Parabacteroides in Bacteroidetes $(\mathrm{p}=0.038)$ and Odoribacter in Bacteroidetes ( $\mathrm{p}=0.008)$, but a lower abundance of Parasutterella in Proteobacteria ( $\mathrm{p}=0.015)$, Marvinbryantia in Firmicutes $(\mathrm{p}<0.001)$ and Turicibacter in Firmicutes ( $\mathrm{p}=0.001)$. The copy number for three of these five genera were further altered by ADR treatment in $\mathrm{BALB} / \mathrm{C}$ mice, but was similar in $\mathrm{C} 57 \mathrm{BL} / 6$ mice before and after treatment. In BALB/C mice, Turicibacter was significantly decreased by $75.7 \%(\mathrm{p}=0.006)$ and Marvinbryantia by $74.0 \%(\mathrm{p}=0.024)$, while Odoribacter was increased around fourfold $(\mathrm{p}<0.001)$. Besides, Rikenella was also significantly decreased by $98.5 \%$ $(\mathrm{p}=0.041)$.

\section{Altered gut microbiome associated with proteinuria}

Since ADR led to the distinct gut microbiome alternation in BALB/C mice with renal damage, an association between increased proteinuria and altered gut microbiome was proposed as viable. We then analyzed three genera, which were different between two kinds of mice and changed after ADR treatment in BALB/C mice (Fig. 4). Results showed that Marvinbryantia $(\mathrm{r}=-0.773$, $\mathrm{p}=0.003)$, Odoribacter $(\mathrm{r}=0.723, \mathrm{p}=0.008)$ and Turicibacter $(\mathrm{r}=0.941, \mathrm{p}<0.001)$ were directly associated with the levels of UACR. Multiple regression analysis about the three microbiome and UACR revealed that Turicibacter is independently associated with the levels of $\operatorname{UACR}\left(\mathrm{R}^{2}=0.898, \mathrm{p}<0.001\right)$.

Furthermore, we detected the serum IL-2 concentration in BALB/C and C57BL/6 mice (Fig. 4d). Two strains presented similar serum levels of IL-2 at baseline $(\mathrm{p}=0.481)$. ADR exposure obviously increased the levels of IL-2 in BALB/C mice $(\mathrm{p}<0.001)$, but not in C57BL/6 mice $(p=0.623)$. These data suggested that increased IL-2 levels might be involved in the process of ADR treatment in BALB/C mice, while the stable levels of IL-2 in C57BL/6 mice were parallel to their resistance to ADR.

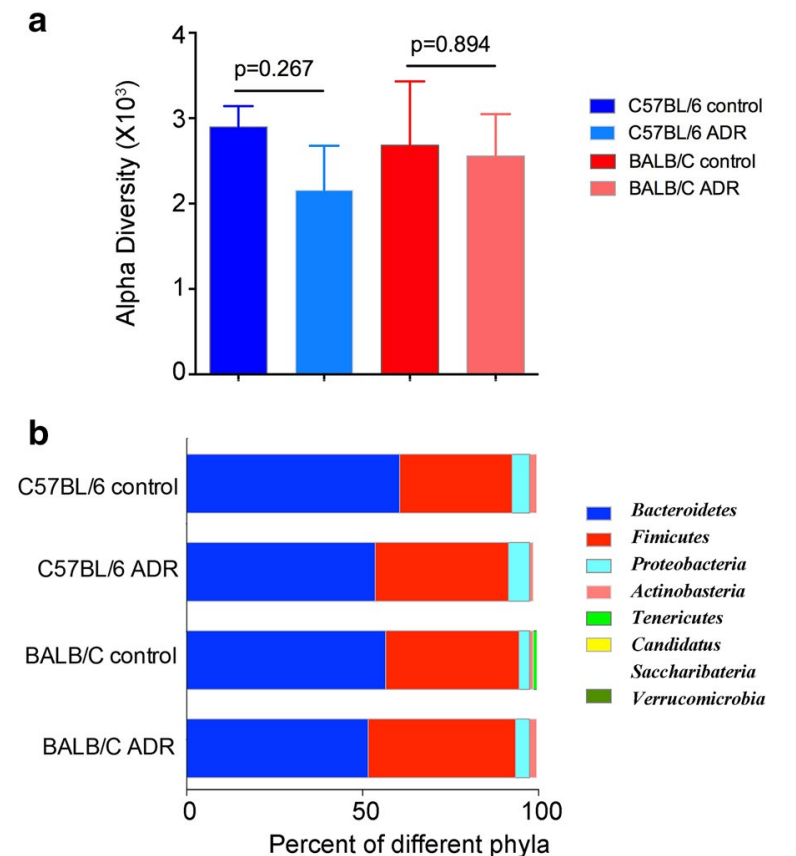

Fig. 2 Gut microbiome altered with ADR at phylum level. a The alpha diversity in the control and ADR group of different mice. $\mathbf{b}$ The gut microbiome composition profiles at the phylum level

\section{Discussion}

In the present study, we confirmed that BALB/C mice are sensitive to ADR-induced nephropathy, while C57BL/6 mice are resistant to such damage. We also showed the distinct gut microbiome and its association with renal damage between BALB/C and C57BL/6 mice. IL-2 was further indicated as being involved in the process of ADR treatment, although the underlying mechanism is far from clear. To the best of our knowledge, this study is the first report indicating the contribution of certain altered gut genus for the increased UACR in an animal model.

Bertani et al. (1982) reported that ADR can induce nephrotic syndrome with morphologic changes in the kidney. Jeansson et al. (2009) suggested that ADR induces direct cell toxic damage to the glomerulus with subsequent tubulointerstitial injury. Lee and Harris (2011) further showed that the early nephrotoxic effect of ADR was related to a generation of oxygen free radicals. Since ADR-induced nephropathy mimicking human proteinuric disease, it is widely used in the study of pathogenesis of renal damage as well as in pharmacology (Cheng et al. 2007; Guo et al. 2008; Wu et al. 2014). However, ADR-induced nephropathy has been found to be species dependent. C57BL/6 mice are normally an ADR resistant strain, while BALB/C mice are an ADR susceptible strain (Sun et al. 2013). We confirmed this species difference in the present study. Until now, the underlying 


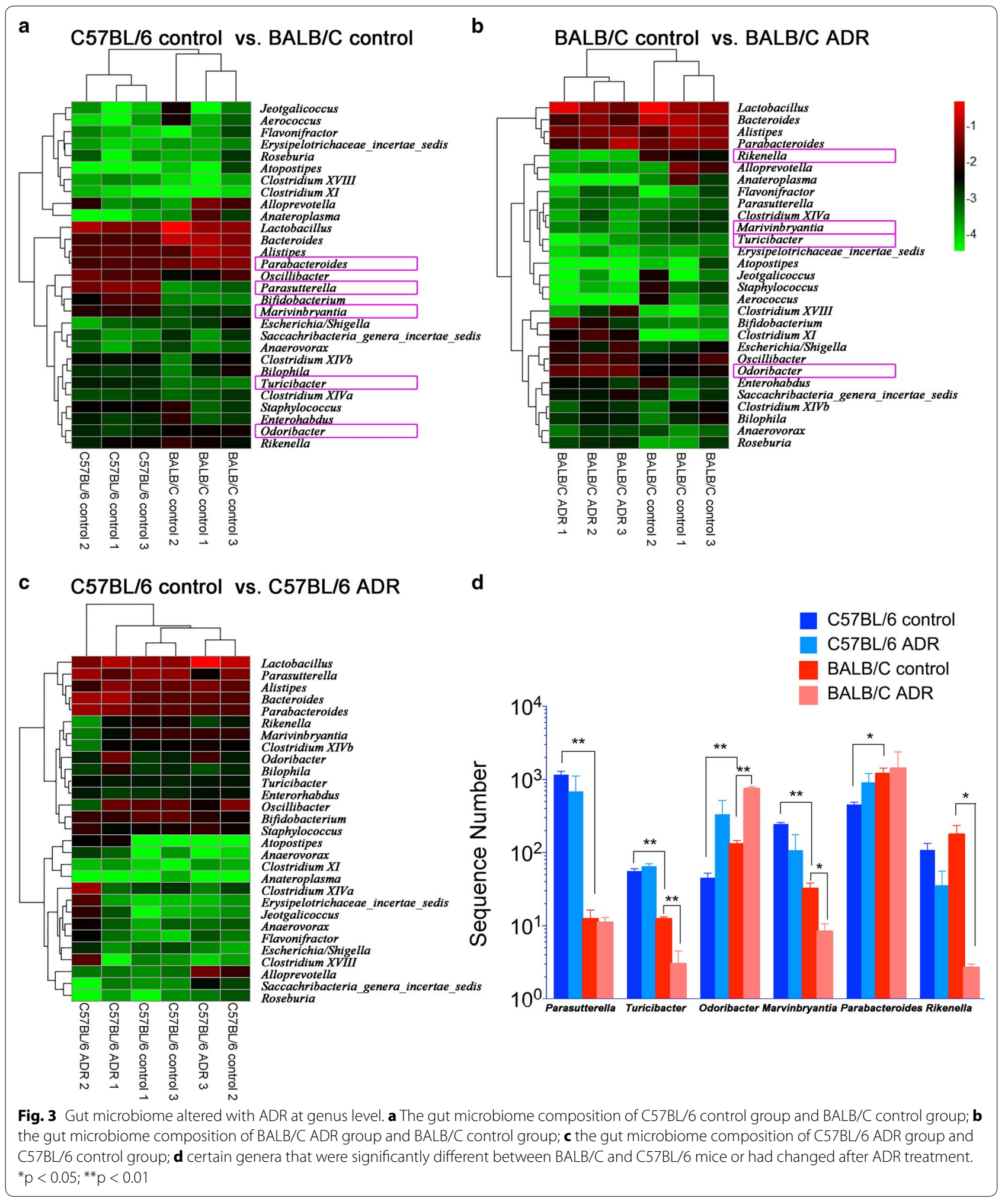

mechanism have not been substantiated, but we have provided a hypothesis about the possible mechanism for the different reaction between these 2 species of mice.
For BALB/C mice, ADR treatment influenced the diversity and function of the gut microbiota with implications for health. Disturbance of the gut flora, referred to as 'gut 

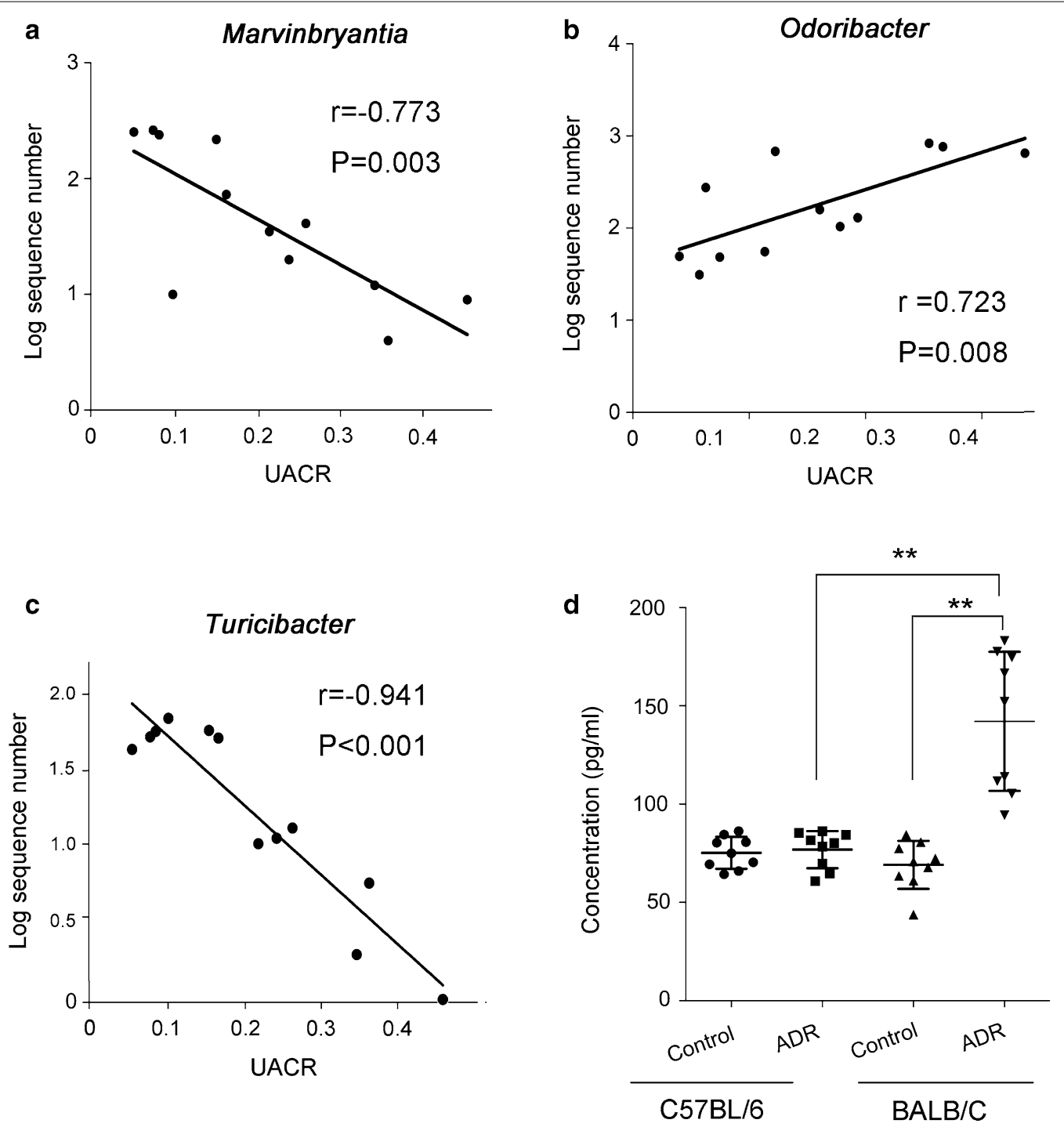

Fig. 4 The association between the microbiomes and the UACR. a The association between the Marvinbryantia and the UACR. $\mathbf{b}$ The association between the Odoribacter and the UACR. c The association between the Turicibacter and the UACR. $\mathbf{d}$ The serum IL-2 concentrations of the four groups

dysbiosis,' (i.e., the reduction of Turicibacter and Marvinbryantia, and the increase of Odoribacter) induced a disruption of the epithelial barrier, ultimately resulting in an increased serum IL-2 level. The renal toxicity came from the metabolites and an association were found between the gut microbiome alteration and the urinary albumin/creatinine ratio (UACR). While for ADR resistant C57BL/6 mice, there were no significant alteration of the gut microbiota after ADR treatment. Unaffected gut microbiota may avoid a disruption of the epithelial barrier and following disease. To support this hypothesis about the mechanism, future researches should demonstrated that restoration of gut microbial composition in
BALB/C mice treated with ADR (e.g., by means of probiotic or fecal transplant therapy) would improve albuminuria and/or inflammation.

In this study explored the difference of gut microbiome composition and reaction to the ADR treatment, and found that it might prompt the distinct renal response. C57BL/6 mice demonstrated a distinct profile of microbiome file in gut. For BALB/C mice, ADR induced obvious proteinuria with a significant change in gut microbiome, and their copy number was directly associated with proteinuria. However, C57BL/6 mice were resistant to ADR treatment and absent from kidney damage and gut microbiome alteration. ADR, as an anthracycline 
antibiotic, is widely used in solid and hematopoietic malignancy therapies. It is considered as one of the simplest and most effective chemotherapeutic agents (Liu et al. 2013), while several significant side effects are also reported, such as ADR induced cardiomyopathy, typhlitis and proteinuria (Chen et al. 2015; Pestalozzi et al. 1993). ADR has also been known to induce gastrointestinal epithelial damage (Fahim et al. 2011) and alter the composition of gut microbiota, which induces the translocation of bacteria species and then stimulates immune responses (Viaud et al. 2013). These finding support the association between gut microbiome and proteinuria under ADR treatment in certain species.

Gut microbiome phyla composition was similar among different mice, which is also in consistent with previous reports (Sommer and Backhed 2013). The ADR treatments led to a decreased pattern of alpha diversity, suggesting a light poison exposure (Zhang et al. 2015). In the model of BALB/C mice, we illustrated the significance of Marvinbryantia, Odoribacter and Turicibacter and highlighted the independent effect of Turicibacter associated with UACR.

Turicibacter was reported to be involved in antiinflammatory properties (Presley et al. 2010; Suchodolski et al. 2012). It has been verified that the levels of Turicibacter decrease with idiopathic inflammatory bowel diseases (IBD) in dog models (Suchodolski et al. 2012); while they increase in colitis-resistant mice when compared to those sensitive to IBD (Presley et al. 2010). In this study, we demonstrated that Turicibacter was at a lower abundance in BALB/C mice than in C57BL/6 mice and it even decreases after ADR treatment. We also showed a significant negative correlation between Turicibacter and proteinuria. Considering the relationship between inflammation and kidney damage, our data supported the possible anti-inflammatory effect of Turicibacter in the mice model of mice treated with ADR. The significance of Odoribacter and Marvinbryantia was also demonstrated in this model.

Odoribacter was described to contribute to the inflammatory process and be involved in IBD, Crohn's disease and ulcerative colitis (Stenvinkel 2005). Our study found that Odoribacter was higher in BALB/C mice, and it even increased after ADR treatment, indicating a direct association of Odoribacter and proteinuria. Little research has mentioned Marvinbryantia, which was lower in $\mathrm{BALB} / \mathrm{C}$ mice than in C57BL/6 mice and even decreased after ADR treatment in this study.

In addition, cytokine IL- 2 is often considered be correlated with gut microbiome level (Bajaj et al. 2012). Certain families, including Alcaligeneceae, Porphyromonadaceae and Enterobacteriaceae, were strongly associated with IL-2 levels in patients with hepatic encephalopathy
(Bajaj et al. 2012). Increasing functions of IL-2 have proven to be resistant to microorganism infection and regulation of Tregs growth, survival and activity (Ohkura et al. 2011), which play a role in IBD (Waidmann et al. 2002), cirrhosis (Bajaj et al. 2012), and itchy psoriasis (Reich and Szepietowski 2007). Although the underlying mechanism is not clear, our data showed the association of IL-2 with ADR-induced proteinuria. The serum IL-2 obviously increased with ADR exposure in BALB/C mice. Parallel to the resistance to ADR-induced proteinuria, the serum IL-2 had no significant change after ADR treatment in C57BL/6 mice.

Thus, our findings suggested a new insight to understanding the ADR-induced proteinuria and established the relationship between the gut microbiome and the proteinuria in vivo. Although we could not present a detailed report showing the effect of gut microbiome on the kidney, we did show the difference of response to ARD treatment between BALB/C and C57BL/6 mice and provided a possible mechanism. This research revealed kidney damage, microbiome alteration and a level of inflammation due to ADR treatment. The reason for the different responses of microbiome to ADR treatment is still a mystery. More investigation is also needed to clarify the detail mechanism of increased IL-2 involved in kidney damage and microbiome alteration.

\section{Abbreviations \\ ADR: Adriamycin; UACR: urinary albumin/creatinine ratio; IL: levels of inter- leukin; CKD: chronic kidney disease; ESRD: end stage renal disease; IS: indoxyl sulphate; PCS: $p$-cresyl sulphate; NS: normal saline; CREA: creatinine; CCr: creatinine clearance; PCR: polymerase chain reaction; OTUs: the operational taxonomic units; RDP: Ribosomal Database Project; ANOVA: one-way analysis of variance; IBD: inflammatory bowel diseases.}

\section{Authors' contributions}

$\mathrm{HL}$ and $\mathrm{HC}$ conceived and designed the study. $\mathrm{Q}, \mathrm{XH}, \mathrm{YZ}$, and $\mathrm{YD}$ performed the experiments. QJ, XH and YZ wrote the paper. QJ, XH, YZ, HL and HC reviewed and edited the manuscript. All authors read and approved the final manuscript.

\section{Author details \\ ${ }^{1}$ Orthodontic Department, Nanjing Stomatological Hospital, Medical School of Nanjing University, Nanjing, Jiangsu, China. ${ }^{2}$ State Key Laboratory of Pol- Iution Control and Resource Reuse, School of the Environment, Nanjing University, Nanjing, Jiangsu, China. ${ }^{3}$ Institute of Medical Genetics, Medical School of Nanjing University, Nanjing, Jiangsu, China. ${ }^{4}$ Jiangsu Key Laboratory of Molecular Medicine, Department of Medical Genetics, Nanjing University, Nanjing, Jiangsu, China.}

\section{Acknowledgements}

Thanks Carla Roberts for her professional writing services.

\section{Competing interests}

The authors declare that they have no competing interests.

Availability of data and materials

All data are fully available without restriction.

Consent for publication

Not applicable. 


\section{Ethics approval and consent to participate}

All applicable international, national, and/or institutional guidelines for the care and use of animals were followed. All animal study protocols were approved by the Vanderbilt University Institutional Animal Care and Use Committee. This article does not contain any studies with human participants performed by any of the authors.

\section{Funding}

This work was supported in part by National Natural Science Foundation (NSF) Grants 81611130131 and 81570714 (to H.C.).

\section{Publisher's Note}

Springer Nature remains neutral with regard to jurisdictional claims in published maps and institutional affiliations.

Received: 1 January 2017 Accepted: 8 December 2017

Published online: 28 February 2018

\section{References}

Bajaj JS, Ridlon JM, Hylemon PB, Thacker LR, Heuman DM, Smith S, Sikaroodi M, Gillevet PM (2012) Linkage of gut microbiome with cognition in hepatic encephalopathy. Am J Physiol Gastrointest Liver Physiol 302:G168-G175. https://doi.org/10.1152/ajpgi.00190.2011

Bertani T, Poggi A, Pozzoni R, Delaini F, Sacchi G, Thoua Y, Mecca G, Remuzz G, Donati MB (1982) Adriamycin-induced nephrotic syndrome in rats: sequence of pathologic events. Lab Invest 46:16-23

Chen S, Chen J, Huang P, Meng XL, Clayton S, Shen JS, Grayburn PA (2015) Myocardial regeneration in Adriamycin cardiomyopathy by nuclear expression of GLP1 using ultrasound targeted microbubble destruction. Biochem Biophys Res Commun 458:823-829. https://doi.org/10.1016/j. bbrc.2015.02.038

Cheng H, Wang S, Jo Yl, Hao CM, Zhang M, Fan X, Kennedy C, Breyer MD, Moeckel GW, Harris RC (2007) Overexpression of cyclooxygenase-2 predisposes to podocyte injury. J Am Soc Nephrol 18:551-559. https:// doi.org/10.1681/asn.2006090990

Eknoyan G, Lameire N, Barsoum R, Eckardt KU, Levin A, Levin N, Locatelli F, MacLeod A, Vanholder R, Walker R, Wang H (2004) The burden of kidney disease: improving global outcomes. Kidney Int 66:1310-1314. https:// doi.org/10.1111/j.1523-1755.2004.00894.x

Evenepoel P, Poesen R, Meijers B (2016) The gut-kidney axis. Pediatr Nephrol. https://doi.org/10.1007/s00467-016-3527-x

Fahim MA, Kataya H, El-Kharrag R, Amer DA, al-Ramadi B, Karam SM (2011) Ghrelin attenuates gastrointestinal epithelial damage induced by doxorubicin. World J Gastroenterol 17:3836-3841. https://doi.org/10.3748/wjg. v17.i33.3836

Guo J, Ananthakrishnan R, Qu W, Lu Y, Reiniger N, Zeng S, Ma W, Rosario R, Yan SF, Ramasamy R, D'Agati V, Schmidt AM (2008) RAGE mediates podocyte injury in Adriamycin-induced glomerulosclerosis. J Am Soc Nephrol 19:961-972. https://doi.org/10.1681/asn.2007101109

Hsu CC, Yang HT, Ho JJ, Yin MC, Hsu JY (2016) Houttuynia cordata aqueous extract attenuated glycative and oxidative stress in heart and kidney of diabetic mice. Eur J Nutr 55:845-854. https://doi.org/10.1007/ s00394-015-0994-y

Jeansson M, Bjorck K, Tenstad O, Haraldsson B (2009) Adriamycin alters glomerular endothelium to induce proteinuria. J Am Soc Nephrol 20:114-122. https://doi.org/10.1681/asn.2007111205

Joshi NA, Fass JN (2011) Sickle: a sliding-window, adaptive, quality-based trimming tool for FastQ files (Version 1.33) [Software]. https://github.com/ najoshi/sickle

Lee VW, Harris DC (2011) Adriamycin nephropathy: a model of focal segmental glomerulosclerosis. Nephrology (Carlton) 16:30-38. https://doi. org/10.1111/j.1440-1797.2010.01383.x

Liu S, Jia Z, Zhou L, Liu Y, Ling H, Zhou SF, Zhang A, Du Y, Guan G, Yang T (2013) Nitro-oleic acid protects against Adriamycin-induced nephropathy in mice. Am J Physiol Renal Physiol 305:F1533-F1541. https://doi. org/10.1152/ajprenal.00656.2012

Liu MC, Lee YW, Lee PT, Chang CS, Tai YL, Yu JR, Su XT, Hsu LW, Lin SH, Wu CH, Liu PY (2015) Cyclophilin A is associated with peripheral artery disease and chronic kidney disease in geriatrics: the Tianliao Old People (TOP) study. Sci Rep 5:9937. https://doi.org/10.1038/srep09937

Martinez-Castelao A, Gorriz JL, Bover J, Segura-de la Morena J, Cebollada J, Escalada J, Esmatjes E, Facila L, Gamarra J, Gracia S, Hernandez-Moreno J, Llisterri-Caro JL, Mazon P, Montanes R, Morales-Olivas F, Munoz-Torres M, de Pablos-Velasco P, de Santiago A, Sanchez-Celaya M, Suarez C, Tranche $S$ (2014) Consensus document for the detection and management of chronic kidney disease. Semergen 40:441-459. https://doi.org/10.1016/j. semerg.2014.09.001

Ohkura N, Hamaguchi M, Sakaguchi S (2011) FOXP3+ regulatory T cells: control of FOXP3 expression by pharmacological agents. Trends Pharmacol Sci 32:158-166. https://doi.org/10.1016/j.tips.2010.12.004

Okuda S, Oh Y, Tsuruda H, Onoyama K, Fujimi S, Fujishima M (1986) Adriamycin-induced nephropathy as a model of chronic progressive glomerular disease. Kidney Int 29:502-510

Pestalozzi BC, Sotos GA, Choyke PL, Fisherman JS, Cowan KH, O'Shaughnessy JA (1993) Typhlitis resulting from treatment with taxol and doxorubicin in patients with metastatic breast cancer. Cancer 71:1797-1800

Presley LL, Wei B, Braun J, Borneman J (2010) Bacteria associated with immunoregulatory cells in mice. Appl Environ Microbiol 76:936-941. https:// doi.org/10.1128/aem.01561-09

Reich A, Szepietowski JC (2007) Mediators of pruritus in psoriasis. Mediators Inflamm 2007:64727. https://doi.org/10.1155/2007/64727

Sandsmark DK, Messe SR, Zhang X, Roy J, Nessel L, Lee Hamm L, He J, Horwitz EJ, Jaar BG, Kallem RR, Kusek JW, Mohler ER 3rd, Porter A, Seliger SL, Sozio SM, Townsend RR, Feldman HI, Kasner SE (2015) Proteinuria, but not eGFR, predicts stroke risk in chronic kidney disease: chronic renal insufficiency cohort study. Stroke 46:2075-2080. https://doi.org/10.1161/ strokeaha.115.009861

Sommer F, Backhed F (2013) The gut microbiota-masters of host development and physiology. Nat Rev Microbiol 11:227-238. https://doi.org/10.1038/ nrmicro2974

Stenvinkel P (2005) Inflammation in end-stage renal disease-a fire that burns within. Contrib Nephrol 149:185-199. https://doi.org/10.1159/000085525

Suchodolski JS, Markel ME, Garcia-Mazcorro JF, Unterer S, Heilmann RM, Dowd SE, Kachroo P, Ivanov I, Minamoto Y, Dillman EM, Steiner JM, Cook AK, Toresson $L$ (2012) The fecal microbiome in dogs with acute diarrhea and idiopathic inflammatory bowel disease. PLoS ONE 7:e51907. https://doi. org/10.1371/journal.pone.0051907

Sun YB, Qu X, Zhang X, Caruana G, Bertram JF, Li J (2013) Glomerular endothelial cell injury and damage precedes that of podocytes in Adriamycininduced nephropathy. PLoS ONE 8:e55027. https://doi.org/10.1371/ journal.pone.0055027

Vaziri ND, Wong J, Pahl M, Piceno YM, Yuan J, DeSantis TZ, Ni Z, Nguyen TH, Andersen GL (2013) Chronic kidney disease alters intestinal microbial flora. Kidney Int 83:308-315. https://doi.org/10.1038/ki.2012.345

Viaud S, Saccheri F, Mignot G, Yamazaki T, Daillere R, Hannani D, Enot DP, Pfirschke C, Engblom C, Pittet MJ, Schlitzer A, Ginhoux F, Apetoh L, Chachaty E, Woerther PL, Eberl G, Berard M, Ecobichon C, Clermont D, Bizet C, Gaboriau-Routhiau V, Cerf-Bensussan N, Opolon P, Yessaad N, Vivier E, Ryffel B, Elson CO, Dore J, Kroemer G, Lepage P, Boneca IG, Ghiringhelli F, Zitvogel L (2013) The intestinal microbiota modulates the anticancer immune effects of cyclophosphamide. Science 342:971-976. https://doi. org/10.1126/science.1240537

Waidmann M, Allemand Y, Lehmann J, di Genaro S, Bucheler N, Hamann A, Autenrieth IB (2002) Microflora reactive IL-10 producing regulatory $T$ cells are present in the colon of IL-2 deficient mice but lack efficacious inhibition of IFN-gamma and TNF-alpha production. Gut 50:170-179

Wang Y, Wang YP, Tay YC, Harris DC (2000) Progressive Adriamycin nephropathy in mice: sequence of histologic and immunohistochemical events. Kidney Int 58:1797-1804. https://doi.org/10.1046/j.1523-1755.2000.00342.x

Wu JB, Ye SF, Liang CL, Li YC, Yu YJ, Lai JM, Lin H, Zheng J, Zhou JY (2014) Qi-Dan Fang ameliorates Adriamycin-induced nephrotic syndrome rat model by enhancing renal function and inhibiting podocyte injury. J Ethnopharmacol 151:1124-1132. https://doi.org/10.1016/j.jep.2013.12.028

Zhang Y, Zhao F, Deng Y, Zhao Y, Ren H (2015) Metagenomic and metabolomic analysis of the toxic effects of trichloroacetamide-induced gut microbiome and urine metabolome perturbations in mice. J Proteome Res 14:1752-1761. https://doi.org/10.1021/pr5011263 